\title{
Is Waist-to-Height Ratio Superior to Body Mass Index and Waist Circumference in Predicting the Incidence of Hypertension?
}

\author{
Na Li ${ }^{a, b}$ Tian Yang ${ }^{a, b}$ Wen-Qian Yu ${ }^{a, b}$ Hao Liu ${ }^{a, b}$ \\ a Department of Obstetrics and Gynecology, Shengjing Hospital of China Medical University, Shenyang, PR \\ China; ${ }^{b}$ Liaoning Centre for Prenatal Diagnosis, Key Laboratory of Maternal-Fetal Medicine of Liaoning Province, \\ Shenyang, PR China
}

\section{Keywords}

Body mass index $\cdot$ Incident hypertension - Waist

circumference $\cdot$ Waist-to-height ratio $\cdot$ Waist-to-hip ratio

\begin{abstract}
Background: It is still controversial which anthropometric indicator could be the best predictor of the incident hypertension. Objectives: To examine the relative power of body mass index (BMI), waist circumference (WC), skinfold thickness, waist-to-hip ratio (WHR), and waist-to-height ratio (WHTR) in predicting the incidence of hypertension in Chinese adults. Method: Data were obtained from the China Health and Nutrition Survey. Overweight was defined as BMI $\geq 23 \mathrm{~kg} / \mathrm{m}^{2}$ and general obesity as BMI $\geq 27.5 \mathrm{~kg} / \mathrm{m}^{2}$. Abdominal obesity was defined by WC values $\geq 90 \mathrm{~cm}$ for males and $\geq 80 \mathrm{~cm}$ for females. Skinfold thickness, WHR, and WHTR were divided into low and high groups according to receiver operating characteristics. Cox regressions and nomograms were employed to compare the relative power of 5 indicators in predicting incident hypertension. Results: When all indicators were analyzed simultaneously, the best predictor of incident hypertension was general obesity $(p<0.001$, adjusted hazard ratio [HR] 1.9, 95\% Cl 1.6-2.2). The results strat-
\end{abstract}

ified by sex showed that BMI and WC were the more powerful predictors of hypertension in males (adjusted HR 1.8 and $1.3,95 \% \mathrm{Cl} 1.4-2.3$ and $1.1-1.5$, respectively) as well as in females (adjusted HR 2.0 and 1.4, 95\% Cl 1.6-2.4 and 1.2-1.6, respectively). Conclusions: $\mathrm{BMI}$ and $\mathrm{WC}$ may predict incident hypertension better than skinfold thickness, WHR, and WHTR in the Chinese population.

(c) 2019 S. Karger AG, Basel

\section{Introduction}

Hypertension has been considered one of the major contributing factors to the burden of diseases all over the world. Overall, the prevalence of hypertension is about $25 \%$ in adults, but this value is expected to increase to $29 \%$ by 2025 [1]. In China, by contrast, the prevalence of hypertension in adults rose from $14.5 \%$ in 1991 to $34.0 \%$ in $2012[2,3]$. Therefore, hypertension has become the major public health issue in the Chinese population.

It has been established that there is a strong association of general obesity with hypertension [4]. As the standard measure of general obesity, body mass index (BMI), is the most widely used indicator to predict and screen hyper-

\section{KARGER}

(c) 2019 S. Karger AG, Basel

E-Mail karger@karger.com

www.karger.com/anm 
tension [5]. Since fat distribution is more important than total body fat for cardiovascular diseases (CVD) $[6,7]$, many measures considering body fat distribution, such as waist circumference (WC), waist-to-hip ratio (WHR), waist-to-height ratio (WHTR), and subcutaneous fat like triceps skinfold thickness, have been developed and applied. In recent decades, many studies have been conducted to investigate the associations of different obesity indicators with hypertension. Some studies indicated that WC or WHTR might be better predictors for CVD risk $[8,9]$. However, other studies reported that WC was the best indicator in Cameroonians, Greek, or Japanese [10, 11]. The American Diabetes Association declared that it is not clear whether WC, compared to BMI, could more precisely predict cardiovascular risk [12]. Some studies based on Japanese and Cuban populations suggested that BMI was the best single indicator of hypertension [13, 14]. Meanwhile, some systematic reviews and meta-analyses have been conducted to examine the power of common obesity indicators in predicting hypertension. Some of those supported that WHTR was a better predictor of CVD risk $[15,16]$, while another meta-analysis concluded that WC was a better predictor and should be recommended to be used in clinic and research [17]. Therefore, it remains contentious and controversial, which is the best predictor of hypertension.

Therefore, in this prospective cohort study, the aim of this study was to evaluate the power of BMI, WC, skinfold thickness, WHR, and WHTR in predicting the incidence of hypertension in the Chinese population.

\section{Materials and Methods}

\section{Study Design}

Data were obtained from the China Health and Nutrition Survey (CHNS), which is an ongoing, open-cohort, international, collaborative project between the Carolina Population Center at the University of North Carolina at Chapel Hill and the National Institute for Nutrition and Health (formerly the National Institute of Nutrition and Food Safety) of the Chinese Center for Disease Control and Prevention (CCDC). This CHNS aimed at exploring how the social and economic transformation of Chinese society has affected the health and nutritional status of the Chinese population, by evaluating the effects of health, nutrition, and family planning policies and programs implemented by national and local governments. The CHNS covers 9 provinces, which vary substantially in geography, economic development, public resources, and health indicators. A multistage random cluster process was employed to sample in each province. Counties in the 9 provinces were stratified by income (low, middle, and high), and a weighted sampling scheme was used to select 4 counties per province at random. Since 1989, 9 rounds of the CHNS were conducted subsequently in 1989,
1991, 1993, 1997, 2000, 2004, 2006, 2009, and 2011. A detailed description of the survey design and procedures has been published elsewhere [18].

\section{Study Population}

Data from 7 waves of the CHNS conducted from 1993 to 2011 were included in this study. Subjects aged 18 years or older at baseline and with data on age, sex, and detailed physical examination (e.g., weight, height, WC, skinfold thickness, hip circumference $[\mathrm{HC}]$, systolic blood pressure [SBP], and diastolic blood pressure [DBP]) were included. The following subjects were excluded: those who were pregnant or lactating at the time of survey, or who were with missing data or implausible outlying data (e.g., weight $>300 \mathrm{~kg}$ or $<20 \mathrm{~kg}, \mathrm{WC}<20 \mathrm{~cm}$, WHR or WHTR $>1$ ), or who were with SBP $\geq 140 \mathrm{~mm} \mathrm{Hg}$ or DBP $\geq 90 \mathrm{~mm} \mathrm{Hg}$, or who took antihypertensive medications, or who self-reported a diagnosis of hypertension at baseline.

\section{Measurement and Definition of Indicators}

Weight, height, and WC were measured by trained healthcare workers following standardized protocols, as set by the World Health Organization (WHO) [19]. Height was measured to the nearest $0.1 \mathrm{~cm}$ without shoes using a portable stadiometer, and weight was measured to the nearest $0.1 \mathrm{~kg}$ with lightweight clothing using a calibrated beam scale. BMI was calculated as weight in kilograms divided by the square of height in meters. WC was measured at a point midway between the lowest rib and the iliac crest in a horizontal plane using non-elastic tape, and HC was measured at the level of the maximum extension of the buttocks posteriorly in a horizontal plane with the participants wearing light clothes and arms open sideways. WHR was calculated as WC (cm) divided by $\mathrm{HC}(\mathrm{cm})$, and WHTR was calculated as WC $(\mathrm{cm})$ divided by height $(\mathrm{cm})$. Skinfold thickness was measured using skinfold calipers and recorded to the nearest $0.5 \mathrm{~mm}$ at the triceps on the right arm (between the tip of the olecranon process of the ulna and the acromion process of the scapula). Three measurements were taken for all indicators, and the averages were used for further analyses. Smoking and drinking status, as well as physical activity were recorded for each subject using a questionnaire. The CHNS asked subjects for their current smoking status and a value of "1" was assigned to those who were currently smoking and a value of " 0 " to those who did not smoke at the time of survey or never smoked. The drinking status was defined in the same way. Physical activity was defined as no ( $<1 \mathrm{~h}$ per week) or yes (one or more hours per week).

Blood pressure measurements were taken after rest for $10 \mathrm{~min}$ in the seated position, with $30 \mathrm{~s}$ intervals between cuff inflations, using standard mercury sphygmomanometers [20]. SBP and DBP were recorded as the points at which the first and fifth Korotkoff sounds appeared respectively. The average of 3 measurements was used. SBP and DBP were recorded in each wave and used to identify whether subjects developed new onset hypertension.

According to the WHO recommendations for Chinese people, overweight was defined as $\mathrm{BMI} \geq 23 \mathrm{~kg} / \mathrm{m}^{2}$ and general obesity as $\mathrm{BMI} \geq 27.5 \mathrm{~kg} / \mathrm{m}^{2}$. In addition, abdominal obesity was defined by $W C$ values $\geq 90 \mathrm{~cm}$ for males and $\geq 80 \mathrm{~cm}$ for females [21]. According to the receiver operating characteristic curve, skinfold thickness, WHR, and WHTR were divided into low and high groups by cut-off points as following: in males: triceps skinfold thickness = $7 \mathrm{~cm}$ with area under curve $(\mathrm{AUC})=0.5117, \mathrm{WHR}=0.8571$ with 
$\mathrm{AUC}=0.5498$, and WHTR $=0.4748$ with $\mathrm{AUC}=0.5811$; in females: triceps skinfold thickness $=23.67 \mathrm{~cm}$ with $\mathrm{AUC}=0.4885$, WHR $=0.8247$ with AUC $=0.5792$, and WHTR $=0.4818$ with AUC $=0.6219$. Hypertension was defined as SBP/DBP $\geq 140 / 90 \mathrm{~mm}$ $\mathrm{Hg}$, or use of hypertensive medications, or a self-reported diagnosis $[20,22]$.

\section{Statistical Analysis}

According to the normality tests, data were reported as medians (inter quartile ranges) for continuous abnormal variables and frequencies (percentages) for categorical variables. Baseline characteristics were compared between non-hypertension and hypertension groups by Wilcoxon rank sum test for continuous abnormal variables and by chi-square tests for categorical variables. Cox regressions were stratified by sex, with hypertension as the outcome and the time interval between the baseline and hypertension diagnosis as the time variable. The censored outcomes were from 2 groups: (i) those who were not diagnosed with hypertension until either drop-out from the cohort or the end of the study (2011); and (ii) those who were not diagnosed with hypertension until death prior the end of the study. To correct for the competing risks of death due to hypertension, all models were adjusted for death. In adjusted models, age, sex, smoking, drinking, physical activity, and ethnic at baseline were adjusted. According to the regression parameter of each factor, nomogram was employed to illustrate the score of each factor, which was used to calculate the probability of developing hypertension. A nomogram is a graphical representation of a mathematical model involving several predictors to predict a particular endpoint based on traditional statistical method such as Cox proportional hazards model for survival data [23]. Nomograms have become very popular tools among clinicians. A step by step guide for building, interpreting, and using nomograms to estimate the hazard function of a particular failure time can be found in a previous study [23]. Nomograms create a simple graphical representation of a statistical predictive model mapping each predictor to a point scale. The predicted probability of the event for a patient by accumulating the total points corresponding to the specific configuration of covariates for that patient. Nomograms have been shown to have high accuracy and discriminating ability for predicting outcomes. The calculation of a Cox regression nomogram is as following: Step (1) Get the scores for all variables values, Step (2) Add the scores = Total score, Step (3) Calculate the probability of survival for a given number of time units given the Total score. The score of each indicator points to the contributor to the dependent variable. And the probability of the outcome at a particular failure time was calculated according to the total score. All analyses were conducted using SAS 9.4 (SAS Institute Inc., Cary, NC, USA). $p \leq 0.05$ with 2 -tailed test was taken as statistical significance.

\section{Results}

There were 10,648 subjects with a median age of 38.5 years. Of these, 3,717 subjects were affected by hypertension during the 18-year follow-up period. There were significant differences between non-hypertension and hypertension groups in all characteristics of the base- line except height $(p=0.491)$. Compared to the non-hypertension group, the hypertension group tended to be older, higher in weight, BMI, WC, WHR, and WHTR, and more likely to be male, Han nationality, smoking, drinking, and physical inactivity (Table 1).

Results of the Cox regressions showed that when BMI, WC, skinfold thickness, WHR, and WHTR were analyzed separately, all of them were significant predictors of hypertension (all $p<0.001$ ). General obesity was the strongest predictor of hypertension (hazard ratio [HR] 3.1, 95\% CI 2.8-3.6). The results, when adjusted for covariates, were consistent with crude results. When the 5 indicators were analyzed together, significant effects were observed for all of them (all $p<0.001$ ). However, when adjusted for covariates, the significance of WHR disappeared $(p=0.169)$. The best predictor of incident hypertension was also general obesity $(p<0.001$, adjusted HR 1.9, 95\% CI 1.6-2.2). These results are presented in Table 2.

The results of the Cox regressions stratified by sex are shown in Table 3. In males, BMI, WC, skinfold thickness, WHR, and WHTR were the significant predictors of incident hypertension (all $p<0.001$ ) when analyzed as single indicators. General obesity and WC were more powerful to predict hypertension (HR 3.1 and 2.3, 95\% CI $2.5-3.8,2.0-2.7$, respectively). The results were comparable before and after adjustment for covariates. When 5 indicators were entered into model 2 simultaneously, all indicators remained statistically significant (all $p<0.001$ ) except WHR ( $p=0.059)$. When adjusted for covariates, BMI (adjusted HR 1.8, 95\% CI 1.4-2.3) was comparable with WC (adjusted HR 1.3, 95\% CI 1.1-1.5) and skinfold thickness (adjusted HR 1.3, 95\% CI 1.2-1.4) but better than WHTR (adjusted HR 1.2, 95\% CI 1.1-1.4).

For the female subsample, BMI, WC, skinfold thickness, WHR, and WHTR were significant predictors of incident hypertension whether or not adjusting for covariates (all $p<0.001$ ) when the 5 indicators were analyzed separately. When all indicators were entered into a model simultaneously, the significance of skinfold thickness disappeared $(p=0.734)$. However, BMI and WC, with adjusting for covariates, were statistically significant predictors of the incidence of hypertension (all $p<0.001$, adjusted HR 1.4, 2.0, and 1.4, 95\% CI 1.2-1.5, 1.6-2.4, $1.2-1.9$ respectively) but skinfold thickness, WHR, and WHTR were not $(p=0.388,0.795$, and 0.199 respectively). General obesity was the strongest predictor of incident hypertension.

Nomograms of Cox regressions were used to obtain scores, which were used to calculate the probability of de- 
Table 1. The characteristics of all subjects at the baseline

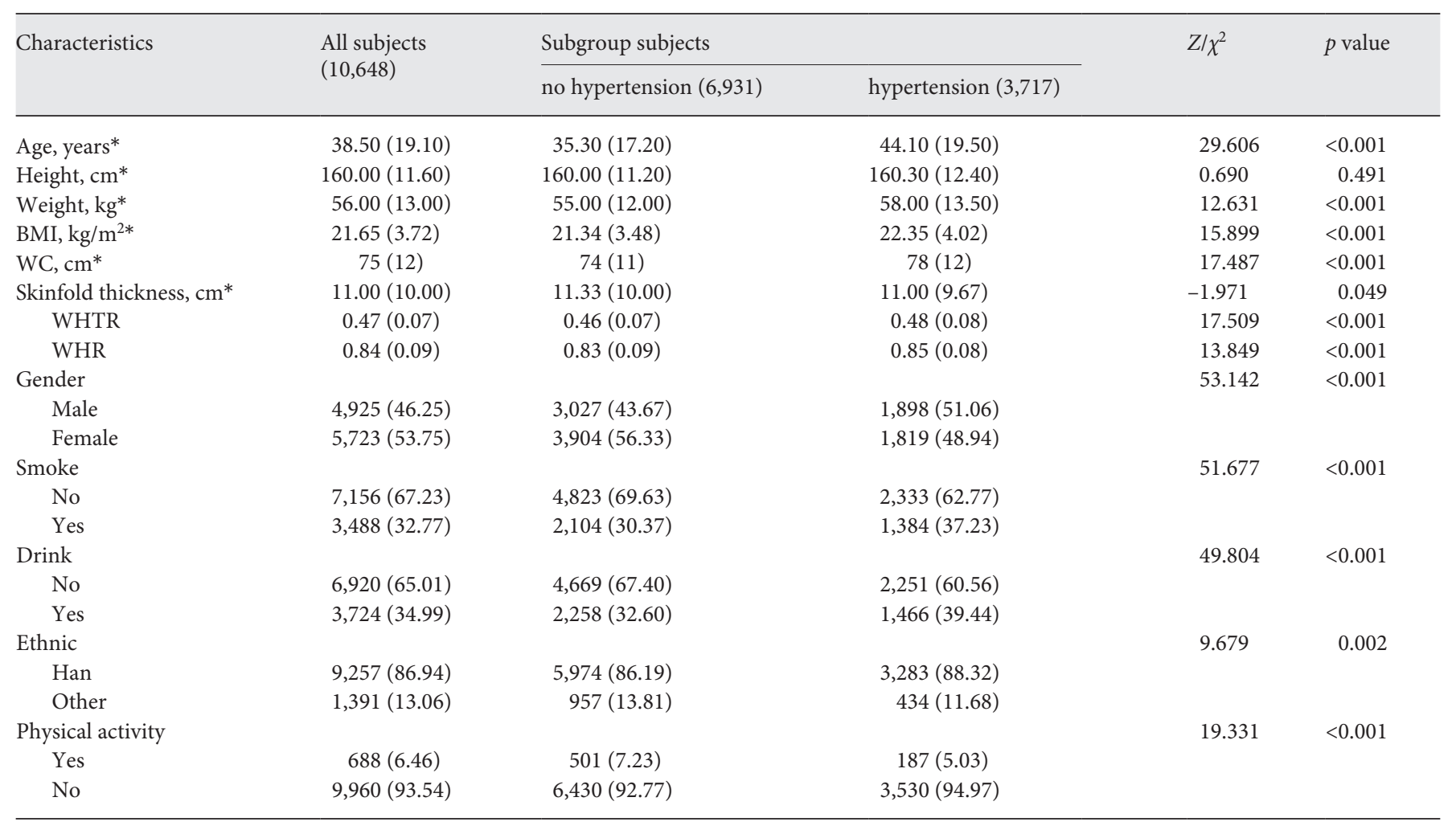

* These variables were analyzed using Wilcoxon rank sum test and $Z$ statistic.

BMI, body mass index; WC, waist circumference; WHTR, waist-to-height ratio; WHR, waist-to-hip ratio.

Table 2. The associations of different anthropometric indicators and the incidence of hypertension from Cox regressions

\begin{tabular}{|c|c|c|c|c|c|c|c|c|c|c|}
\hline \multicolumn{11}{|l|}{ BMI, $\mathrm{kg} / \mathrm{m}^{2}$} \\
\hline Overweight & 0.6 & 267.915 & $<0.001$ & 1.8 & $1.7-1.9$ & 0.5 & 214.232 & $<0.001$ & 1.7 & $1.6-1.8$ \\
\hline Obesity & 1.1 & 295.106 & $<0.001$ & 3.1 & $2.8-3.6$ & 1.0 & 240.733 & $<0.001$ & 2.8 & $2.5-3.2$ \\
\hline WHR & 0.5 & 215.570 & $<0.001$ & 1.6 & $1.5-1.8$ & 0.3 & 90.923 & $<0.001$ & 1.4 & $1.3-1.5$ \\
\hline WHTR & 0.7 & 453.727 & $<0.001$ & 2.1 & $1.9-2.2$ & 0.5 & 224.041 & $<0.001$ & 1.7 & $1.6-1.8$ \\
\hline \multicolumn{11}{|l|}{ Model $2^{\#}$} \\
\hline \multicolumn{11}{|l|}{$\mathrm{BMI}, \mathrm{kg} / \mathrm{m}^{2}$} \\
\hline Overweight & 0.2 & 25.661 & $<0.001$ & 1.3 & $1.1-1.4$ & 0.3 & 45.752 & $<0.001$ & 1.4 & $1.2-1.5$ \\
\hline Obesity & 0.5 & 42.205 & $<0.001$ & 1.7 & $1.4-2.0$ & 0.6 & 58.880 & $<0.001$ & 1.9 & $1.6-2.2$ \\
\hline
\end{tabular}

* In models 1 and 2, sex, age, smoke, drink, physical activity and ethnic were adjusted for.

\# In model 1, all indicators were analyzed separately with low as the reference. In model 2, 5 indicators were entered into model simultaneously. BMI, body mass index; WC, waist circumference; WHR, waist-to-hip ratio; WHTR, waist-to-height ratio. 
Table 3. The associations of different anthropometric indicators and the incidence of hypertension by sex from Cox regressions

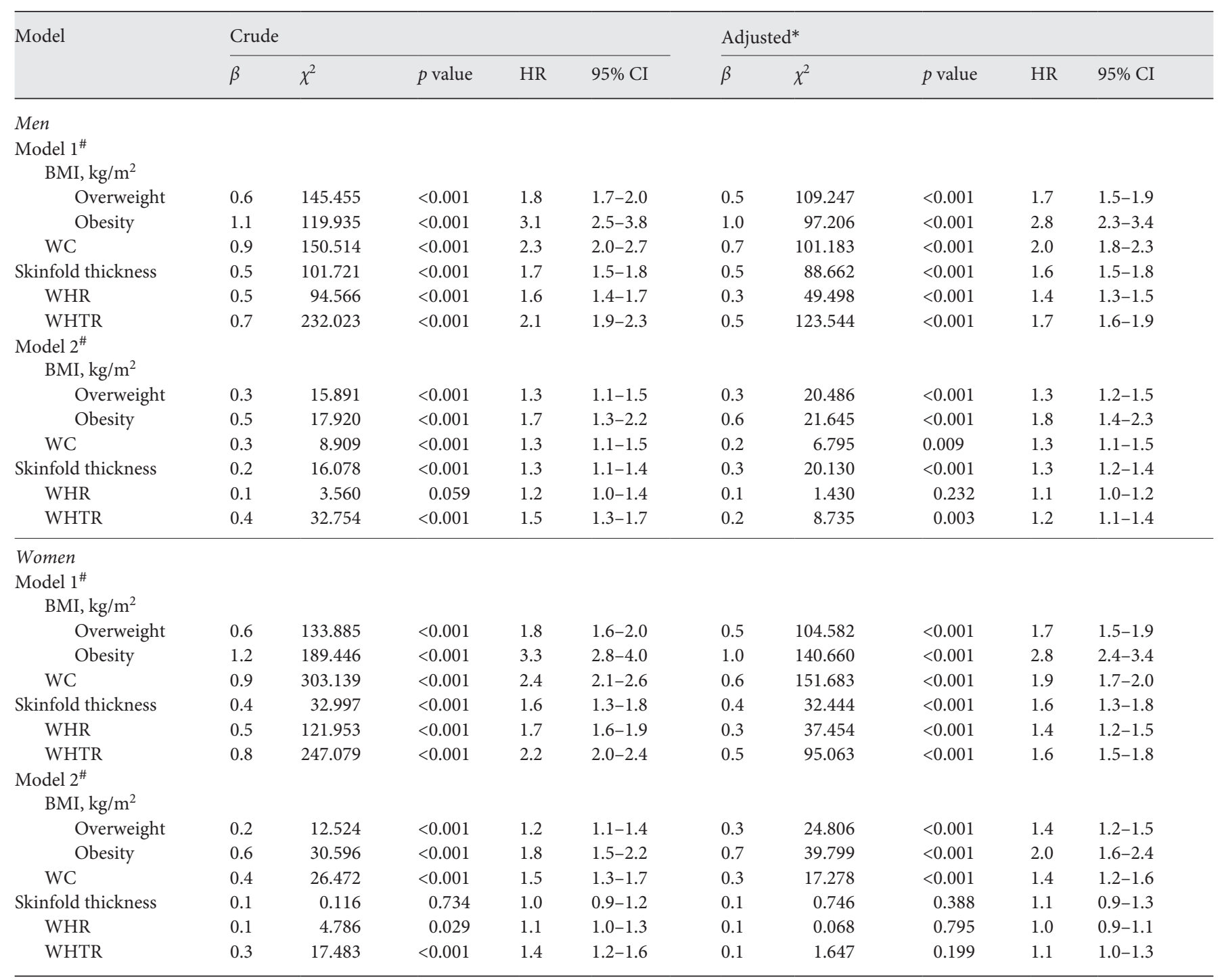

* In models 1 and 2, age, smoke, drink, physical activity and ethnic were adjusted for.

\# In model 1, all indicators were analyzed separately with low as the reference. In model 2, 5 indicators were entered into model simultaneously. BMI, body mass index; WC, waist circumference; WHR, waist-to-hip ratio; WHTR, waist-to-height ratio.

veloping hypertension given certain time. The scores for BMI were higher than those for other indicators in both sexes as shown in Figures 1 and 2.

\section{Discussion}

In the 18-year follow-up study of 10,648 Chinese men and women, BMI, WC, skinfold thickness, WHR, and WHTR were the significant predictors of incident hypertension when they were analyzed separately. However, general obesity might be the best predictor of hypertension when 5 indicators entered into a model simultaneously, especially when adjusting for covariates. Moreover, compared to other predictors, BMI and WC were more powerful and effective to predict incident hypertension in males as well as in females.

Up to date, there was no consistent conclusion on the relative power and effectiveness of different anthropometrics in predicting the incidence of hypertension. Some studies reported that BMI or WC was the best predictor of incident hypertension [11, 13, 14, 24]. While 


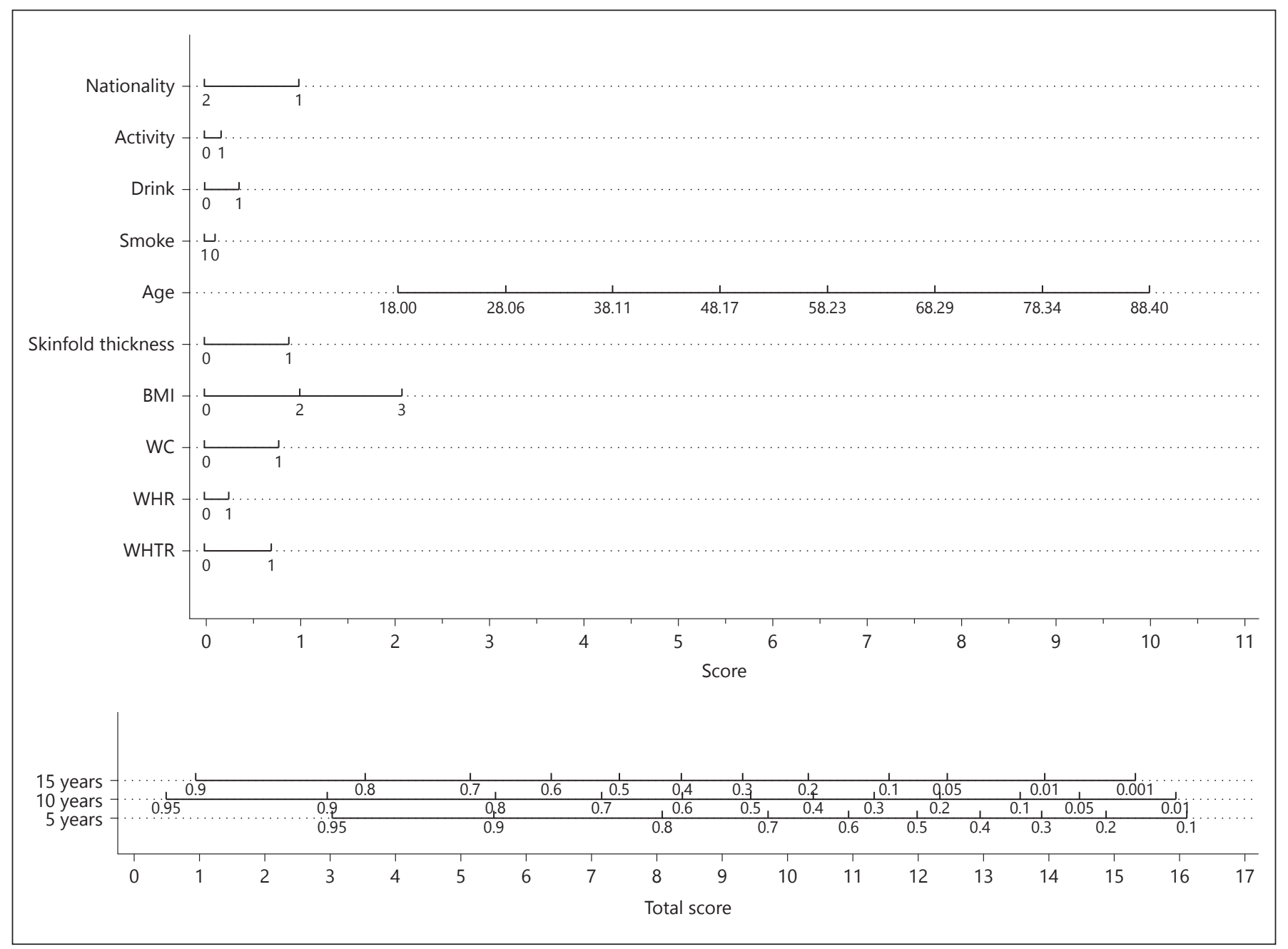

Fig. 1. The Cox nomogram of obesity-related indicators to predict the incidence of hypertension in males. Notably, the details of scores calculation by nomogram were as follows: Step (1) Establish scores for all variable values: Nationality $=1=>$ Score $\approx$ 1 ; Activity $=1=>$ Score $\approx 0.25$; Drink $=1=>$ Score $\approx 0.45$; Smoke $=1=>$ Score $\approx 0$; Age (years) $=18=>$ Score $\approx 2.1$; Skin fold thickness $=1=>$ Score $\approx 1$; $\mathrm{BMI}=2=>$ Score $\approx 1$; WC $=$ $1=>$ Score $\approx 0.8 ;$ WHR $=1=>$ Score $\approx 0.2 ;$ WHTR $=1=>$

others declared that WHTR was superior to predict the incidence of hypertension $[6,25,26]$. In the present study, BMI and WC, as the classical general and abdominal obesity indicators, were superior to predict the development of hypertension in Chinese men and women, which was consistent with previous studies [5, 27, 28]. Moreover, the performance of BMI and WC decreased when a combination of them was entered into a model. This implied that BMI and WC should be simultaneously but independently used to predict the incidence of hypertension.
Score $\approx 0.7$. Step (2) Obtain the total score by adding up all the scores obtained in the previous step. Total score $=1+0.25+$ $0.45+0+2.1+1+1+0.8+0.2+0.7=7.5$. Step (3) Obtain the probability of event (Total Score -> Probability of event). Total score $=7.5$ is equivalent to a probability of approximately 0.50 when the failure time was 15 years. BMI, body mass index; WC, waist circumference; WHR, waist-to-hip ratio; WHTR, waist-toheight ratio.

$\mathrm{BMI}$ and WC were confirmed to be more powerful to predict the incident hypertension in the present study. Differences across studies might be attributed to variations in population characteristics, sampling strategies, the quality of data collection, and the differences in operational definitions of general obesity and abdominal obesity [28]. For example, a previous study reported that Asians were smaller in HC than Americans [29]. Moreover, the present study population was followed for 18 years. With the development of economy and society, BMI and WC might obviously change during the follow-up pe- 


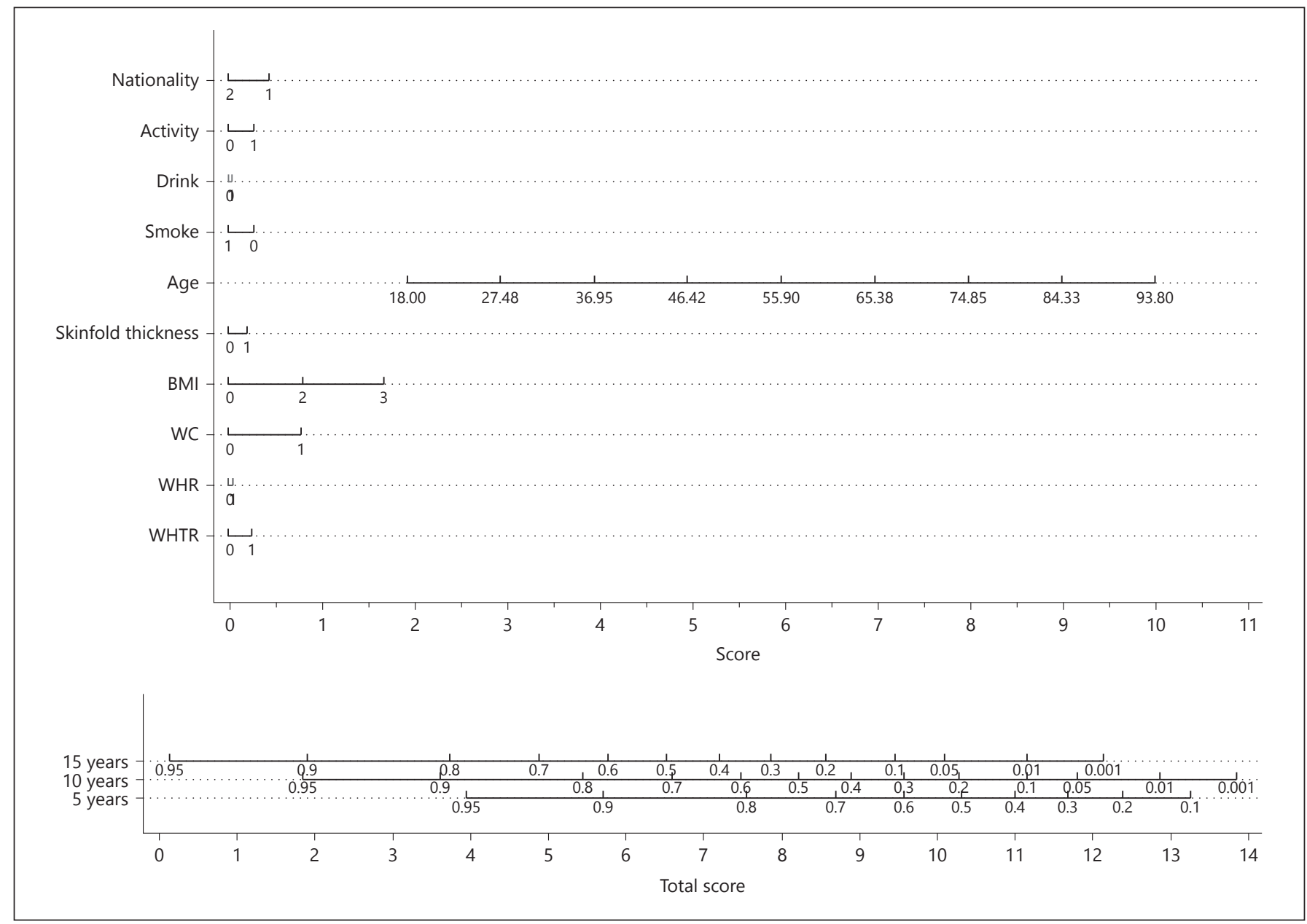

Fig. 2. The Cox nomogram of obesity-related indicators to predict the incidence of hypertension in females. BMI, body mass index; WC, waist circumference; WHR, waist-to-hip ratio; WHTR, waist-to-height ratio.

riod, and more and more people tended to be with higher body fat mass. But the definitions of general obesity and abdominal obesity remain unchanged. Thus, more subjects would be affected by general obesity and abdominal obesity. Therefore, compared to other anthropometric indicators, the application of BMI and WC might improve the identification of incident hypertension.

In this study, nomogram was employed to calculate the score of each risk factor, which was used to predict the probability of developing hypertension. In both males and females, the scores of BMI were the highest among all anthropometrics, which indicated that BMI was the best predictor of incident hypertension. Increasing BMI might result in increases in serum glucose, insulin, aldosterone, and renin levels along with increased sympathetic tone. These factors mentioned above were likely to increase blood pressure by increasing vascular volume or peripheral resistance [30].
The combination of BMI, WC, skinfold thickness, WHR, and WHTR was analyzed in a model to compare their relative powers in predicting hypertension. Since there are correlations among WC, WHR, and WHTR, each indicator was divided into low and high groups according to the cutoff points of a receiver operating characteristic. Thus, the correlations of these indicators were considerably reduced when they were entered into a model simultaneously.

\section{Limitations and Strengths}

In this study, data were obtained from a long-term, large-scale, population-based cohort study. Compared to a retrospective study, it would provide more accurate and comprehensive evidence on the comparisons of different obesity-related indicators in predicting incident hypertension. Due to the differences in ethnic and dietary patterns across different countries, the prevalence and extent 
of general obesity were varied. In this study, according to the WHO recommendations for Chinese people, ethnicbased BMI cut-offs were used to define overweight and general obesity. As a result, the misclassification bias was reduced. The nomogram can provide a specific score for each indicator and correct the complex sampling design [31]. The consistent results between nomogram and Cox regressions further confirmed that BMI and WC were more powerful to predict the incident of hypertension. Meanwhile, the limitations of this study should be stated. Since the study population was limited to Chinese men and women, caution should be taken when extrapolating to other ethnic populations. The data on family history of hypertension were not collected in the CHNS. Therefore, this potential confounder could not be corrected for in the analyses. Furthermore, since the nutrients and details of diet intake were not available, the covariates related to hypertension, such as fat and salt intake, failed to be adjusted. Since only triceps skinfold thickness was measured in the CHNS, the comprehensive percent BF failed to be calculated using sum of skinfold thickness at several sites.

In conclusion, WHTR was not superior to BMI and $\mathrm{WC}$ in predicting the incidence of hypertension. By contrast, the powers of BMI and $\mathrm{WC}$ in predicting the incident hypertension were better than skinfold thickness, WHR, and WHTR. The combination of BMI and WC might decrease their performance. Therefore, BMI and WC should be used independently to predict the incidence of hypertension in adults. In the practical application, the better predictor of hypertension should be cautiously chosen according to the characteristics of targeted population and conclusions of local studies.

\section{Acknowledgment}

This research uses data from CHNS. We thank the National Institute of Nutrition and Food Safety, CCDC and Prevention, Carolina Population Center, the University of North Carolina at Chapel Hill, the NIH (R01-HD30880, DK056350, and R01-HD38700) and the Fogarty International Center, NIH for financial support for the CHNS data collection and analysis files from 1989 to 2006 and both parties plus the China-Japan Friendship Hospital, Ministry of Health for support for CHNS 2009 and future surveys.

\section{Ethics Statement}

This study was approved by the Institutional Review Board of the National Institute for Nutrition and Food Safety, CCDC and Prevention, and University of North Carolina at Chapel Hill. All subjects provided the informed consent.

\section{Disclosure Statement}

The authors declare that they have no conflicts of interest to disclose.

\section{Funding Sources}

No funding was received for this study.

\section{Authors Contribution}

N.L.: designed the study and wrote the draft. T.Y.: analyzed the data. W.-Q.Y.: interpreted the results. H.L.: edited and reviewed the manuscript.

\section{References}

1 Mittal BV, Singh AK. Hypertension in the developing world: challenges and opportunities. Am J Kidney Dis. 2010 Mar;55(3):590-8.

2 Li D, Lv J, Liu F, Liu P, Yang X, Feng Y, et al. Hypertension burden and control in mainland China: analysis of nationwide data 2003 2012. Int J Cardiol. 2015 Apr;184:637-44.

3 Ke L, Ho J, Feng J, Mpofu E, Dibley MJ, Li Y, et al. Prevalence, awareness, treatment and control of hypertension in Macau: results from a cross-sectional epidemiological study in Macau, China. Am J Hypertens. 2015 Feb; 28(2):159-65.

4 Chandra A, Neeland IJ, Berry JD, Ayers CR, Rohatgi A, Das SR, et al. The relationship of body mass and fat distribution with incident hypertension: observations from the Dallas Heart Study. J Am Coll Cardiol. 2014 Sep; 64(10):997-1002.
5 Bennasar-Veny M, Lopez-Gonzalez AA, Tauler P, Cespedes ML, Vicente-Herrero T, Yañez A, et al. Body adiposity index and cardiovascular health risk factors in Caucasians: a comparison with the body mass index and others. PLoS One. 2013 May;8(5):e63999.

6 Li WC, Chen IC, Chang YC, Loke SS, Wang $\mathrm{SH}, \mathrm{Hsiao} \mathrm{KY}$. Waist-to-height ratio, waist circumference, and body mass index as indices of cardiometabolic risk among 36,642 Taiwanese adults. Eur J Nutr. 2013 Feb;52(1): 57-65.

7 Janghorbani M, Aminorroaya A, Amini M. Comparison of Different Obesity Indices for Predicting Incident Hypertension. High Blood Press Cardiovasc Prev. 2017 Jun;24(2): 157-66.

8 Park SH, Choi SJ, Lee KS, Park HY. Waist circumference and waist-to-height ratio as predictors of cardiovascular disease risk in Korean adults. Circ J. 2009 Sep;73(9):164350.

9 Zeng Q, He Y, Dong S, Zhao X, Chen Z, Song $Z$, et al. Optimal cut-off values of BMI, waist circumference and waist:height ratio for defining obesity in Chinese adults. Br J Nutr. 2014 Nov;112(10):1735-44.

10 Mbanya VN, Kengne AP, Mbanya JC, Akhtar H. Body mass index, waist circumference, hip circumference, waist-hip-ratio and waistheight-ratio: which is the better discriminator of prevalent screen-detected diabetes in a Cameroonian population? Diabetes Res Clin Pract. 2015 Apr;108(1):23-30.

11 Lee BJ, Kim JY. A comparison of the predictive power of anthropometric indices for hypertension and hypotension risk. PLoS One. 2014 Jan;9(1):e84897. 
12 Gallagher D, Visser M, Sepúlveda D, Pierson RN, Harris T, Heymsfield SB. How useful is body mass index for comparison of body fatness across age, sex, and ethnic groups? Am J Epidemiol. 1996 Feb;143(3):228-39.

13 Sakurai M, Miura K, Takamura T, Ota T, Ishizaki M, Morikawa Y, et al. Gender differences in the association between anthropometric indices of obesity and blood pressure in Japanese. Hypertens Res. 2006 Feb;29(2): $75-80$.

14 Rodrigues Barbosa A, Balduino Munaretti D, Da Silva Coqueiro R, Ferreti Borgatto A. Anthropometric indexes of obesity and hypertension in elderly from Cuba and Barbados. J Nutr Health Aging. 2011 Jan;15(1):17-21.

15 Ashwell M, Gunn P, Gibson S. Waist-toheight ratio is a better screening tool than waist circumference and BMI for adult cardiometabolic risk factors: systematic review and meta-analysis. Obes Rev. 2012 Mar;13(3): 275-86.

16 Savva SC, Lamnisos D, Kafatos AG. Predicting cardiometabolic risk: waist-to-height ratio or BMI. A meta-analysis. Diabetes Metab Syndr Obes. 2013 Oct;6:403-19.

17 van Dijk SB, Takken T, Prinsen EC, Wittink H. Different anthropometric adiposity measures and their association with cardiovascular disease risk factors: a meta-analysis. Neth Heart J. 2012 May;20(5):208-18.

18 Popkin BM, Du S, Zhai F, Zhang B. Cohort Profile: the China Health and Nutrition Survey-monitoring and understanding socioeconomic and health change in China, 19892011. Int J Epidemiol. 2010 Dec;39(6):143540.
19 Eveleth PB. Physical status: the use and interpretation of anthropometry. Report of a WHO Expert Committee. Am J Hum Biol. 1996;8(6):786-7.

20 Chobanian AV, Bakris GL, Black HR, Cushman WC, Green LA, Izzo JL Jr, et al.; Joint National Committee on Prevention, Detection, Evaluation, and Treatment of High Blood Pressure. National Heart, Lung, and Blood Institute; National High Blood Pressure Education Program Coordinating Committee. Seventh report of the Joint National Committee on Prevention, Detection, Evaluation, and Treatment of High Blood Pressure. Hypertension. 2003 Dec;42(6):120652.

21 Expert Consultation WH; WHO Expert Consultation. Appropriate body-mass index for Asian populations and its implications for policy and intervention strategies. Lancet. 2004 Jan;363(9403):157-63.

22 Chobanian AV, Bakris GL, Black HR, Cushman WC, Green LA, Izzo JL Jr, et al.; National High Blood Pressure Education Program Coordinating Committee. The Seventh Report of the Joint National Committee on Prevention, Detection, Evaluation, and Treatment of High Blood Pressure: the JNC 7 report. JAMA. 2003 May;289(19):2560-72.

23 Iasonos A, Schrag D, Raj GV, Panageas KS. How to build and interpret a nomogram for cancer prognosis. J Clin Oncol. 2008 Mar; 26(8):1364-70.

$24 \mathrm{Yu}$ J, Tao Y, Tao Y, Yang S, Yu Y, Li B, et al. Optimal cut-off of obesity indices to predict cardiovascular disease risk factors and metabolic syndrome among adults in Northeast
China. BMC Public Health. 2016 Oct;16(1): 1079.

25 Sayeed MA, Mahtab H, Latif ZA, Khanam PA, Ahsan KA, Banu A, et al. Waist-to-height ratio is a better obesity index than body mass index and waist-to-hip ratio for predicting diabetes, hypertension and lipidemia. Bangladesh Med Res Counc Bull. 2003 Apr;29(1):1-10.

26 Meseri R, Ucku R, Unal B. Waist:height ratio: a superior index in estimating cardiovascular risks in Turkish adults. Public Health Nutr. 2014 Oct;17(10):2246-52.

27 Ononamadu CJ, Ezekwesili CN, Onyeukwu OF, Umeoguaju UF, Ezeigwe OC, Ihegboro GO. Comparative analysis of anthropometric indices of obesity as correlates and potential predictors of risk for hypertension and prehypertension in a population in Nigeria. Cardiovasc J Afr. 2017 Mar/Apr;28(2):92-9.

28 Wai WS, Dhami RS, Gelaye B, Girma B, Lemma S, Berhane Y, et al. Comparison of measures of adiposity in identifying cardiovascular disease risk among Ethiopian adults. Obesity (Silver Spring). 2012 Sep;20(9):1887-95.

29 Li C, Ford ES, Zhao G, Kahn HS, Mokdad AH. Waist-to-thigh ratio and diabetes among US adults: the Third National Health and Nutrition Examination Survey. Diabetes Res Clin Pract. 2010 Jul;89(1):79-87.

30 Wilsgaard T, Schirmer H, Arnesen E. Impact of body weight on blood pressure with a focus on sex differences: the Tromso Study, 19861995. Arch Intern Med. 2000 Oct;160(18): 2847-53.

31 Capanu M, Gonen M. Building a Nomogram for Survey-Weighted Cox Models Using R. 2011. 